\title{
A Testbed for Static Electricity Meter Testing with Conducted EMI
}

\author{
Helko E. van den Brom \\ VSL B.V. \\ Delft, the Netherlands \\ hvdbrom@vsl.nl
}

\author{
Zander Marais \\ VSL B.V. \\ Delft, the Netherlands \\ zmarais@vsl.nl
}

\author{
Dennis Hoogenboom \\ VSL B.V. \\ Delft, the Netherlands \\ dhoogenboom@vsl.nl
}

\author{
Ronald van Leeuwen \\ VSL B.V. \\ Delft, the Netherlands \\ rvleeuwen@vsl.nl
}

\author{
Gert Rietveld \\ VSL B.V. \\ Delft, the Netherlands \\ grietveld@vsl.nl
}

\begin{abstract}
In this paper we present the design and implementation of a new flexible testbed for testing electricity meters with conducted EMI in the frequency range up to $150 \mathrm{kHz}$. This testbed is based on separate generation of voltage and current test signals with arbitrary waveforms. Different transconductance amplifiers were characterized for their suitability to generate the highly-distorted current waveforms required for advanced testing of electricity meters. The complete setup was validated by comparing test results on a meter showing error readings under conducted EMI with earlier test results obtained using a setup based on a power source in combination with real physical loads. The good validation results of the new testbed proof the setup is very suitable for advanced calibration or testing of static electricity meters under wideband conducted EMI. The setup and waveforms presented in this paper will be used as input for improved standardization of electricity meters type testing.
\end{abstract}

Keywords - Static meters, energy measurement, standards, Electromagnetic Compatibility, EMC immunity testing, electricity meters.

\section{INTRODUCTION}

Smart static electricity meters are currently being rolledout over Europe, replacing the familiar electromechanical electricity meters. Recent studies in the Netherlands have shown that some static electricity meters are sensitive to specific conducted electromagnetic interference (EMI), where errors up to several hundreds of percent have been reported $[1][2][3][4]$. The applied loads causing the conducted EMI consisted of the combination of a phase-firing dimmer with a resistive heater or LED and CFL lamps, resulting in chopped or sharply peaked current waveforms with significant frequency content in the range up to around $50 \mathrm{kHz}$. These results suggest that testing according to the presently applied harmonized standards under the EU Measuring Instruments Directive (MID) in Europe might not be sufficient to prove that the static meters being rolled-out fulfill their accuracy limits under all operating conditions.

Critics argue that these waveforms are rather extreme signals that can be generated in the laboratory but do not represent a realistic situation in typical households using CEmarked equipment. After all, problems caused by conducted EMI are known for many years (see, e.g., [5][6][7]), and measures have been taken accordingly by means of EU regulation [8][9] and standardization [10][11].

This work is carried out within the EMPIR project 17NRM02 MeterEMI which has received funding from the EMPIR programme co-financed by the Participating States and from the European Union's Horizon 2020 research and innovation programme.
Triggered by the above-mentioned findings, a European pre-normative research project has recently been started [12]. Equipment is being developed for on-site waveform capturing at residential buildings with suitable waveform analysis algorithms, together with new testbeds for meter testing with the appropriate testing procedures.

Though very important in the discussion whether the laboratory test signals are relevant, real-world waveforms captured on-site at metered supply points do not directly provide us with information on what equipment and what kind of signals exactly cause the observed meter errors. Instead, load current waveforms from different mass-market electrical goods can be captured and categorized according to the relevant key parameters found so far to be responsible for the observed meter errors, i.e., high rate of change of current, peak amplitude and crest factor. Analysis of alternative key parameters, for example based on wavelets or short-time Fourier transforms, is ongoing [13]. For future type testing, from these categorized waveforms representative signals can be selected and, if necessary, modified.

Systematic research and type testing of electricity meters with conducted EMI can only be performed using a testbed that is able to reproduce specific realistic and possibly disturbing conducted EMI waveforms. Current electricity meter testbeds usually sweep a single sine wave over a frequency range from $2 \mathrm{kHz}$ to $150 \mathrm{kHz}$, as specified in [5] and [11]. When performing such measurements at test houses, the undisturbed $50 \mathrm{~Hz}$ signal and the interference signal are usually separately generated and then mixed. For fast edge signals with a rich harmonic content as studied in [1] and [2], the complication is how to synchronize the split signals and to ensure the mixing electronics is sufficiently linear for the waveforms not to become distorted in the time domain.

In this paper we present the development of a new type of testbed able to generate arbitrary waves representing fast switching real-world signals. This new testbed is based on synchronized arbitrary waveform generators in combination with separate voltage and transconductance amplifiers. Measurements will be performed as in [2] and the voltage signal will at first be treated as undisturbed, so the main challenge is to generate and apply the current waveforms with sufficient bandwidth and low distortion in the time domain. For this reason, three different transconductance amplifiers have been investigated. The remainder of this paper will describe the measurement setup in detail, as well as test results for the three different amplifiers and validation results for the final setup. 


\section{MEASUREMENT SETUP}

\section{A. Operating principle}

In normal operation, electricity meters are connected directly to the mains, which provides both voltage and current. The tests described in [1][2][3][4] have been performed this way, with real physical loads connected to the meter under test to define the current waveform. When testing electricity meters, however, the voltage $V$ and current $I$ can be applied separately, without the corresponding power $P=V \cdot I$ being really dissipated. This phantom-power approach is common practice at national metrology institutes for sinusoidal power calibrations and at notified bodies for type testing of electricity meters and will be the approach adopted in this paper.

In the new measurement setup, schematically shown in Fig. 1, the voltage and current signals are generated using an arbitrary waveform generator in combination with a voltage amplifier and a transconductance amplifier, respectively. The current is applied to the phase input and output of the electricity meter under test (labeled L in Fig. 1), whereas the voltage is supplied between its phase input or output and neutral (labeled $\mathrm{N}$ in Fig. 1). The current measurement is performed using a high-precision current shunt in combination with a 2-channel high-precision digitizer used to perform the voltage measurements, which cannot easily be fully decoupled from ground. Therefore, the current shunt needs to be connected in between the Lo output of the transconductance amplifier and the phase input or output of the meter under test. Consequently, the phase connections of the electricity meter are close to ground potential and the neutral senses the full phase voltage of $230 \mathrm{~V}$. This reversal of polarity of the voltage signal is possible because electricity meters are fully floating from ground. Furthermore, the voltage divider, used to sense the voltage signal applied to the meter under test, is connected to input of the isolation transformer, because it measures the voltage with respect to ground potential which is dictated by the digitizer Lo input. Note that for electricity meters in which the voltage and current can be decoupled, this reversal of voltage phase and neutral is not necessary.

From previous work it is known that a highly distorted current waveform with sufficiently high current increase (or time derivative $d I / d t$ ), drawn from a non-ideal power source, causes the voltage waveform to be slightly distorted as well. Nevertheless, in this testbed the voltage signal applied is a sinusoidal waveform of $230 \mathrm{~V}$. The expectation is that this does not have a significant impact on the meter reading.

Present standards, originally developed for electromechanical induction meters, dictate that for testing threephase electricity meters all three phases should be connected. However, the design of static electricity meters is such that the three circuits for the three phases are fully independent. As expected, during our investigations, for static electricity meters we have seen no difference in the results between single-phase and three-phase testing. Therefore, it was decided to connect only a single phase.

\section{B. Description of the equipment used}

To generate the test signals, a sinusoidal voltage and a distorted current waveform are generated using two channels of a NI PXI-6733 8-channel, 16-bit, $1 \mathrm{MS} / \mathrm{s}$ arbitrary

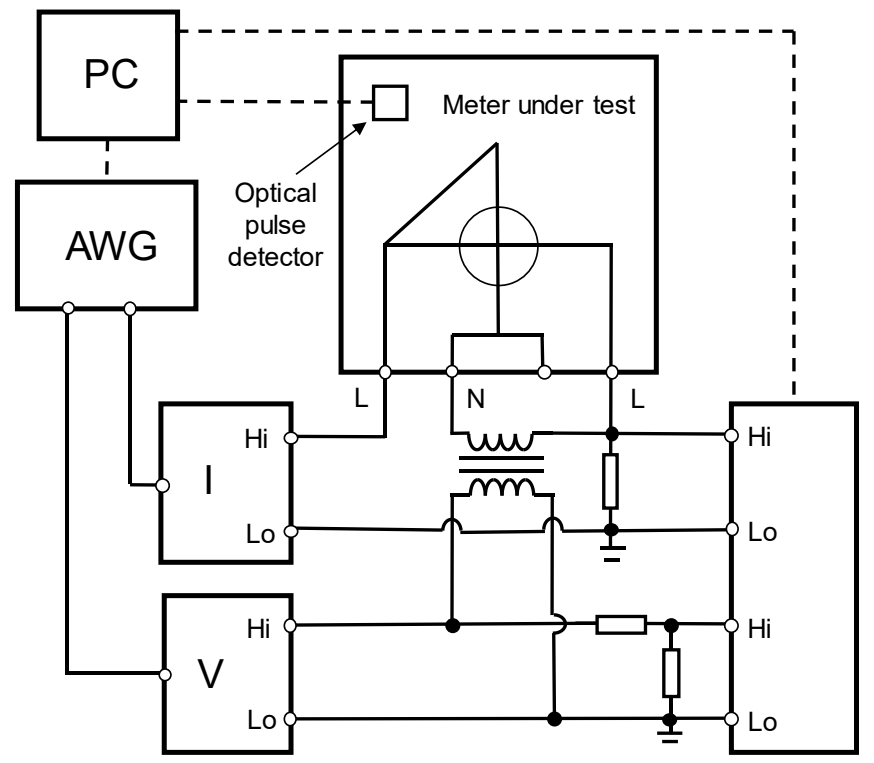

Fig. 1. Schematic of the testbed, with the electricity meter under test which is read out by a PC using an optical pulse detector, a synchronized dual arbitrary waveform generator in combination with voltage and transconductance amplifiers (labeled V and I) and an isolation transformer to generate the test signals, and a current shunt, voltage divider and dual digitizer to measure the test signals.

waveform generator ${ }^{1}$. The corresponding output voltages are fed to a voltage amplifier and a transconductance amplifier, respectively. The voltage amplifier is not critical since it just needs to generate a stable sinusoidal signal, but the transconductance amplifier needs to be able to generate signals with peak currents preferably as high as 100 A without distorting the signal too much in the time domain, i.e., with frequency components up to $150 \mathrm{kHz}$ and sufficiently high slew rates. In this paper we present results comparing three different transconductance amplifiers (specified in the next section) to investigate their suitability for generating the desired current waveforms.

The generated voltage and current signals are continuously measured using calibrated measurement equipment. Hence, traceability of the tests is provided by means of the measurement part of the testbed. The voltage and current measurements are performed using a home-built 150:1 resistive voltage divider and a broadband $50 \mathrm{~m} \Omega$ current shunt [14], respectively, in combination with a NI PXI-5922 2-channel, 24-bit, $15 \mathrm{MS} / \mathrm{s}$ digitizer [15] used at a sampling rate of $1 \mathrm{MS} / \mathrm{s}$. Alternatively, a high-accuracy broadband power meter could be used to measure the effective energy applied to the electricity meter under test.

The energy reading of the meter under test $E_{\mathrm{DUT}}(T)$ is determined via its LED output using an optical pulse detector which is connected to a PC. The time $T$ between two or more pulses is used to calculate the value for the energy consumption according to the reference setup $E_{\mathrm{REF}}(T)$ used in the same time interval $T$ from the measured voltage and current signals $V(t)$ and $I(t)$, respectively,

$$
E_{\mathrm{REF}}(T)=\int_{0}^{T} V(t) \cdot I(t) d t .
$$

Commercial equipment is only identified in this paper to adequately specify the experimental procedure. Such identification does not imply recommendation or endorsement by VSL, nor does it imply that the equipment identified is necessarily the best available for the purpose. 


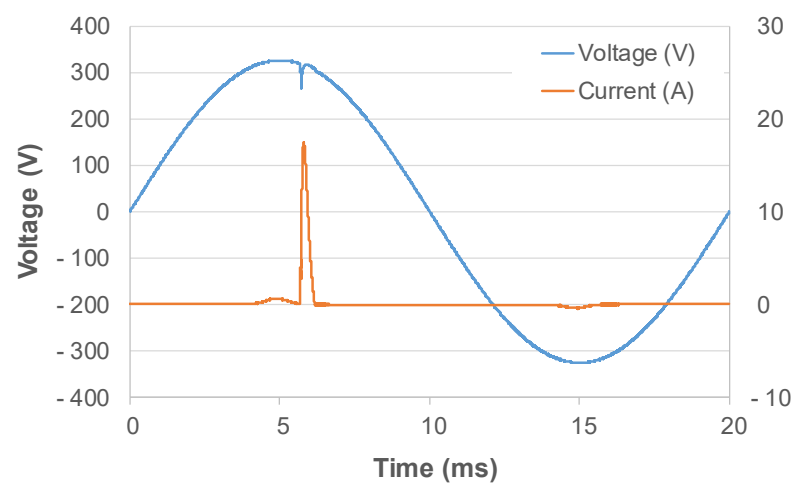

Fig. 2. The applied current test signal (in red) as used for verification of the current waveforms generated by means of the different transconductance amplifiers.

The calculated energy is then compared to the energy consumption $E_{\text {DUT }}(T)$ between the same two pulses according to the meter under test, which is typically one watt-hour per pulse. The error of the meter $\varepsilon$ is defined as the difference between those two energy readings divided by the energy as determined with the reference system,

$$
\varepsilon=\frac{E_{\mathrm{DUT}}(T)-E_{\mathrm{REF}}(T)}{E_{\mathrm{REF}}(T)} .
$$

This error is the key quantity to be determined using the testbed for electricity meters under test.

Note that in the schematic of Fig. 1 the energy consumed by the meter itself is provided by the voltage source and is not observed in the current measurement. Hence, the reference value of the energy does not need to be corrected for the meter consumption, in contrast to the case in which real loads are measured [2]. This is important especially when measuring smaller power levels.

\section{Testing AND VALIDATION MEASUREMENTS}

Testing and validation of the setup is performed in two steps: (1) comparison of the generated current waveforms to the original waveform, and (2) comparing test results of a static meter showing error readings, obtained with the new setup, with the results obtained earlier using the original method with a real load [2]. The validation can be considered complete and the testbed considered suitable for its purpose of advanced static electricity meter testing with highly-distorted current waveforms if the meter readings $E_{\mathrm{DUT}}(T)$ (i.e., their reference value according to Eq. 1 plus their error according to Eq. 2) agree with the original real load test results to within a few percent for all waveforms.

\section{A. Verification of current waveforms}

In the first step, the current waveform as generated using the transconductance amplifier is compared to the applied voltage input waveform. Fig. 2 shows the current waveform chosen for this characterization, generated by a water pump with included dimmer used for fish ponds ${ }^{2}$. This is an extremely distorted signal that results in large error readings of some static meters. Note that the current waveform is unipolar and therefore contains a small DC component. The current signal caused by the electricity meter consumption

\footnotetext{
2. This specific type of water pump is no longer available on the market and has been replaced by a newer version showing less distorted waveforms.
}
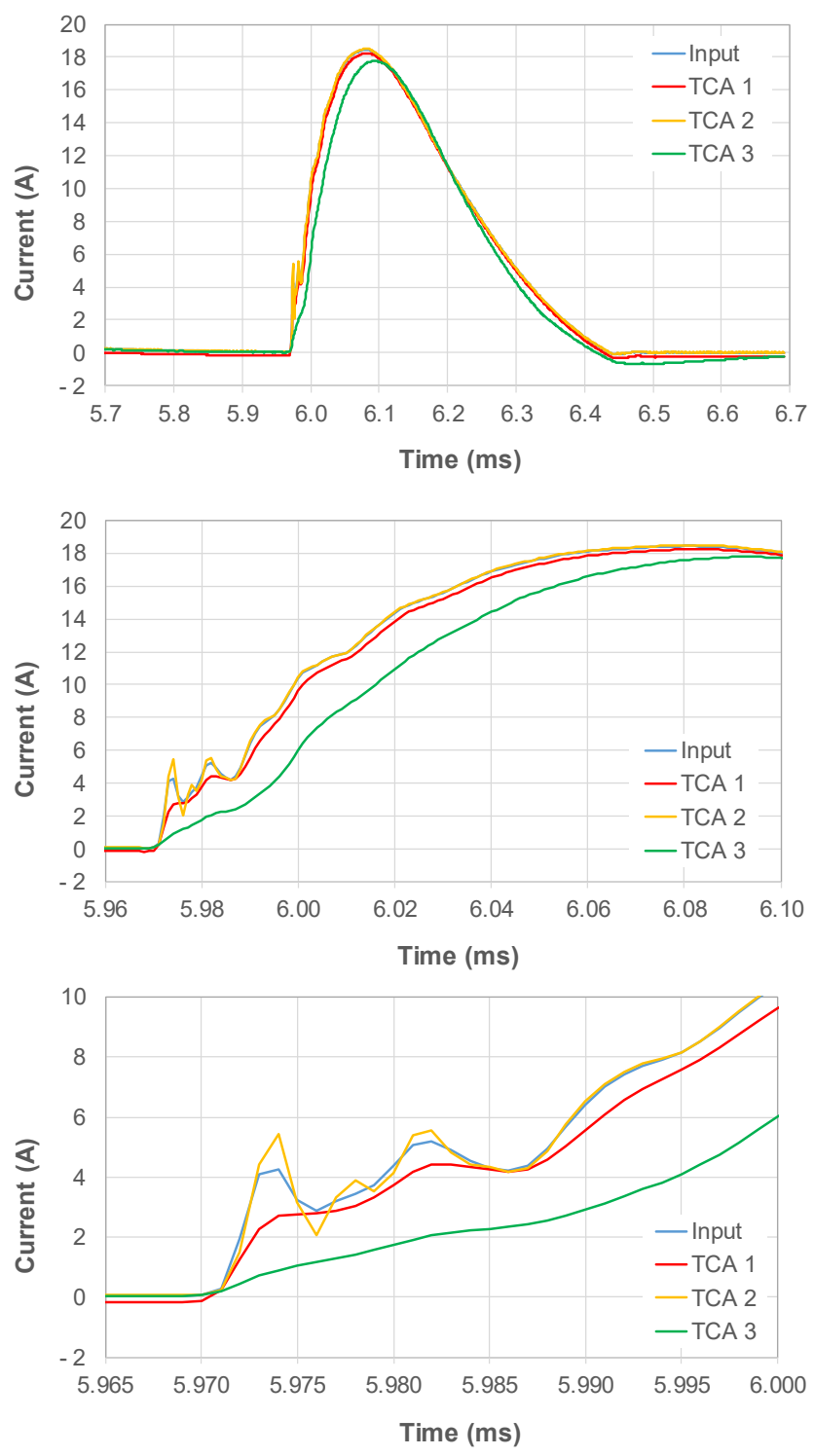

Fig. 3. Current waveforms generated by means of the three different transconductance amplifiers as compared to the (scaled) input voltage waveform.

itself is clearly visible as small bumps around $5 \mathrm{~ms}$ and $15 \mathrm{~ms}$, where the positive and negative maxima of the corresponding voltage waveform occur. Also note that the voltage signal is slightly distorted by the rapidly changing current signal, indicating the limitation of the power source used in the experiment (non-zero output impedance) [2].

Three different transconductance amplifiers have been investigated for their suitability to provide the desired waveform without too much distortion. TCA 1 is an AE Techron 7228 broadband power amplifier with a $1 \mathrm{MHz}$ bandwidth that needs a burden resistance of at least $0.5 \Omega$ and proper tuning. TCA 2 is a Clarke-Hess 8100 broadband highprecision transconductance amplifier with $100 \mathrm{kHz}$ bandwidth, typically used at national metrology institutes. TCA 3 is an older California Instruments $3225 \mathrm{~K}$ broadband power amplifier with similar bandwidth. TCA 1 and TCA 2 can provide current peak amplitudes up to $60 \mathrm{~A}$ and $140 \mathrm{~A}$, respectively, whereas TCA 3 is limited to about $20 \mathrm{~A}$. 
The current waveforms measured at the output of the transconductance amplifiers are compared to the input voltage waveform and to each other in Fig. 3. In this figure, the input voltage is scaled to the corresponding current values as measured in the original setup, in which the current was measured as a voltage over a $50 \mathrm{~m} \Omega$ broadband precision shunt. The upper graph in Fig. 3 shows a time interval of $1 \mathrm{~ms}$ of the current signal around the peak in Fig. 2. The middle graph shows the same graph zoomed in to show the rising edge only, whereas the lower graph is further zoomed to reveal the details of the first current ripple. As can be seen in Fig. 3, none of the transconductance amplifiers is able to perfectly follow the voltage input signal, but clear differences can be observed.

The lower curve in Fig. 3 shows that TCA 1 has a slight DC offset and seems to have limited bandwidth preventing the instrument from following the rapid changes immediately after $280 \mu \mathrm{s}$, when the current starts changing from zero. Similarly, later current variations seem to be somewhat damped as well. The reason for this might be the fact that the burden resistor which was used is limited in bandwidth. In contrast, TCA 2 does not have this offset but seems to have a small overshoot when trying to follow the same rapid changes. For the largest part of the waveform, this amplifier nicely follows the input signal. TCA 3 seems to be unable to follow the rapid changes shown in the lower graph at all, and even seems to have problems returning to zero around $750 \mu \mathrm{s}$, which can be observed in the upper graph. This latter problem may be related to the small DC content of the test signal.

\section{B. Testing of a static electricity meter}

The impact of the characteristics of the different amplifiers on the test results when using the setup described in Fig. 1 was investigated by testing a meter that is known to show error readings when exposed to heavily distorted current signals under real load conditions. The current measurement principle of this meter is based on a Rogowski coil. The test results obtained with the new setup are compared with the results obtained earlier using the original method, in which a power source was used in combination with a real (non-linear) load to generate the currents.

The first verification was to investigate whether indeed the energy consumption of the meter itself of about $2 \mathrm{~W}$ was excluded by the reference system, which is relevant for the test signals corresponding to low power. For this test, zero current was generated, and both the meter and the reference system correctly indicated zero energy, proving that both the meter and the reference system correctly do not measure the energy consumption of the meter itself.

The measurements on the meter under test are performed using a variety of test signals caused by different pieces of household electronics, including a purely resistive heater (labeled R0), the same heater in combination with a phasefiring Toverli TVLX-W-10A+ dimmer set at $75 \%$ (labeled R75), a combination of LED and CFL lamps using the same dimmer at $75 \%$ (labeled CL75), and the earlier mentioned water pump with nominal maximum power of $68 \mathrm{~W}$ with different dimmer settings (labeled WP4 and WP9), one of which was fed directly from the VSL mains instead of a separate power source (labeled WP4-M). Apart from the R0 waveform, which was used for verification purposes only,
TABLE I. METER READING ERRORS FOR DIFFERENT LOADS

\begin{tabular}{cccccc}
$\begin{array}{c}\text { Applied } \\
\text { test signal }\end{array}$ & $\begin{array}{c}\text { Power } \\
\text { (W) }\end{array}$ & $\begin{array}{c}\text { Real load } \\
\text { error (\%) }\end{array}$ & $\begin{array}{c}\text { TCA 1 } \\
\text { error (\%) }\end{array}$ & $\begin{array}{c}\text { TCA 2 } \\
\text { error (\%) }\end{array}$ & $\begin{array}{c}\text { TCA 3 } \\
\text { error (\%) }\end{array}$ \\
\hline \hline R0 & 798 & 0.4 & 0.3 & 0.4 & 0.3 \\
R75 & 148 & 51 & 20 & 46 & -4 \\
CL75 & 297 & 136 & 128 & 142 & $54^{\text {a }}$ \\
WP4 & 34 & 1257 & 1150 & 1261 & $267^{\text {a }}$ \\
WP4-M & 35 & 1947 & 2055 & 2038 & $750^{\text {a }}$ \\
WP9 & 67 & 145 & 132 & 143 & $30^{\text {a }}$ \\
& & \multicolumn{4}{c}{ a. $\quad$ Lower current amplitude used }
\end{tabular}

these waveforms represent a few of the most disturbing household appliances found in our investigations so far.

From earlier experiments we know that the error can be strongly related to the amplitude of the distorted current in a non-linear way. Therefore, in the tests, the amplitude setting of the transconductance amplifiers were tuned such that the energy as determined by the reference system was as close as possible to the energy reading in the original experiments with a real load. For TCA 3, however, the maximum gain is such that to generate $20 \mathrm{~A}$ current a $20 \mathrm{~V}$ input voltage signal is required, which is beyond the $10 \mathrm{~V}$ output range of the waveform generator used in our setup. Therefore, for TCA 3 some of the waveforms have lower amplitudes than for the real load signals, so they cannot directly be compared to the results obtained with TCA 1 and TCA 2 .

The meter errors, defined in Eq. (2) and expressed as a percentage of the energy as determined by the reference system $E_{\mathrm{REF}}(T)$, are given in Table I. In this table, the first column indicates the waveforms used to simulate specific loads, whereas the second column gives the corresponding actual power $E_{\mathrm{REF}}(T)$. The third column provides the meter errors as determined using the original setup with the power source and real physical load. The last three columns show the meter errors obtained with the new setup when using the three different transconductance amplifiers, respectively.

As can be seen from the numbers in the table, the meter errors for TCA 1 show significant deviations for most of the test waveforms. For instance, for the relatively less extreme $\mathrm{R} 75$ waveform, a difference of about $-20 \%$ in meter reading $E_{\text {DUT }}(T)$ (which is related to the meter error by means of Eq. 2 ) as compared to the original load meter reading was observed.

The meter errors when using TCA 2 are very close to the original results with the real load. The largest difference of about $5 \%$ between the meter reading $E_{\mathrm{DUT}}(T)$ when using TCA 2 and the original load meter reading was observed for the WP4-M waveform, which is the one corresponding to the largest meter error.

For TCA 3, apart from the rather trivial R0 waveform, the meter error obtained for the R75 waveform is the only result that could be directly compared to the results obtained with the other amplifiers and with the original results, due to the limitation of the gain setting and output voltage of our waveform generator mentioned before. Nevertheless, for this relatively less extreme R75 waveform, the difference in meter reading $E_{\text {DUT }}(T)$ as compared to the original load meter 
TABLE II. METER READING ERRORS FOR DIFFERENT LOADS AND MEASUREMENT CONFIGURATIONS WHEN USING TCA2

\begin{tabular}{cccc}
$\begin{array}{c}\text { Applied } \\
\text { test signal }\end{array}$ & $\begin{array}{c}\text { Real load } \\
\text { error (\%) }\end{array}$ & $\begin{array}{c}\text { Connected } \\
\text { error (\%) }\end{array}$ & $\begin{array}{c}\text { Disconnected } \\
\text { error (\%) }\end{array}$ \\
\hline R0 & 0.4 & 0.4 & 0.2 \\
R75 & 51 & 46 & 48 \\
CL75 & 136 & 142 & 142 \\
WP4 & 1257 & 1261 & 1251 \\
WP4-M & 1947 & 2038 & 2045 \\
WP9 & 145 & 143 & 140
\end{tabular}

reading was already about $35 \%$, showing that TCA 3 is not suitable for providing the current test signals in our testbed.

Measurements have been performed with the meter under test configured in two settings. The first configuration, corresponding to the one used to obtain the results depicted in Table I, is the conventional one with current and voltage inputs connected. This configuration represents the situation of actual use for metering purposes, which was also used in the original measurements [2], although the grounding is applied differently. In the second configuration, the voltage and current inputs are disconnected, which is usually done for testing purposes.

In Table II, the meter errors obtained for the two measurement configurations when using TCA 2 are compared. The first three columns of Table II are identical to the corresponding ones in Table I, in which the internal meter current and voltage leads were connected. The last column shows the meter errors obtained with the internal meter current and voltage leads disconnected. As can be seen from the numbers in the table, the difference between meter errors obtained with the connected and disconnected measurement configuration is negligible.

\section{DISCUSSION}

The characterization of the transconductance amplifiers presented in Section III.A revealed that none of them was perfect, which is not surprising when generating extreme and highly distorted broadband signals as described in this paper. TCA 2 was selected as the most suitable one because it only showed a small overshoot when programmed to generate a large current step. We expect this small overshoot to be negligible in the tests, and the validation results on a static meter with known errors as described in Section III.B and presented in Table I seem to confirm this assumption. For transconductance amplifier TCA 2, the largest difference in meter reading $E_{\mathrm{DUT}}(T)$ as compared to the original load meter reading was only $5 \%$, which was observed for the waveform corresponding to the largest meter error. For waveforms causing meter errors as large as $2000 \%$ the meter error reading is expected to be very sensitive to small changes in the test signal. Amplifiers TCA 1 and TCA 3 show errors that are too large for them to be used in our testbed. The reason for TCA 1 might be the limited bandwidth of the required $0.5 \Omega$ burden resistor mentioned in section III.A; further finetuning of TCA 1 might be performed in a later stage.

Using the testbed investigated in this paper, the voltage is applied to the electricity meter under test with a polarity different from normal use. That is, the phase or line input $\mathrm{L}$ is close to ground potential, whereas the neutral $\mathrm{N}$ senses almost the full phase voltage of $230 \mathrm{~V}$. Since electricity meters are usually completely floating with respect to ground, one can argue that this voltage polarity reversal should not cause any problem. This assumption is confirmed by the validation measurements described in section III.B and indicated in Table II.

The measurement method used in the new testbed is inherently different from the original one, because in the latter method actual power is dissipated whereas in the former virtually no real power is dissipated. Since the electricity meter under test only measures voltage and current, it should not sense any difference. However, there might be differences between the cases in which the voltage and current leads of the meter under test are internally connected or disconnected. Very small differences have indeed been observed, as shown in Table I. However, this is not completely unexpected, since for these waveforms the current measurement unit of the meter under test is heavily disturbed, possibly because of clipping or overloading, a situation for which the response is not designed to be perfectly reproducible. In such situations, small changes in the measurement configuration can lead to relatively larger differences.

Present standards dictate that for testing three-phase electricity meters a voltage signal should be applied to all three phases. However, these standards are written for conventional electromechanical induction meters, that are expected to be sensitive to the applied voltage on all phases. For the static electricity meters tested at VSL, no such effect has been observed. Therefore, only a single phase needs to be connected in our testbed.

\section{CONCLUSION AND OUTLOOK}

In this paper we presented the design and implementation of a new test facility for static electricity meter testing with conducted EMI representing heavily distorting household equipment. Three different transconductance amplifiers were compared for their ability to reproduce the original waveforms as much as possible. The setup was validated by comparing the test results on a static meter, showing error readings for different waveforms, with the test results obtained with the original setup using a power source and real physical loads. The results of this comparison validate the new testbed using TCA 2 to be a reliable system to test electricity meters for heavily distorted signals in the frequency range up to $150 \mathrm{kHz}$.

Future work will focus on further finetuning of the output of transconductance amplifier TCA 1 , which is a far more reasonably priced instrument as compared to TCA 2. Furthermore, other waveforms and other electricity meters will be investigated and compared to the results obtained using the original setup. Finally, the new setup will be used for future meter type testing purposes.

The waveforms used for testing as described in this paper are beyond the waveforms defined in present meter testing standards [10][11]. Therefore, results of the measurements obtained with this new setup will be presented to standards developing organizations as input for improved standardization of electricity meter type testing. 


\section{ACKNOWLEDGMENT}

The authors thank P.S. Wright and P. Davis from NPL and H. Schouten and H. Boereboom from NMi Certin for valuable discussions.

\section{REFERENCES}

[1] F. Leferink, C. Keyer, A. Melentjev, "Static energy meter errors caused by conducted electromagnetic interference," IEEE Electromagnetic Compatibility Magazine, vol. 5, Issue 4, pp 49-55, Fourth Quarter 2016.

[2] G. Rietveld, D. Hoogenboom and M. Acanski, "Conducted EMI causing error readings of static electricity meters", CPEM Conf. Dig., Paris, France, July 2018.

[3] B. ten Have, T. Hartman, N. Moonen, C. Keyer, F. Leferink, "Faulty readings of static energy meters caused by conducted electromagnetic interference from a water pump", ICREPQ Conf. Dig., Tenerife, Spain, April 2019.

[4] Zander Marais, Helko van den Brom, Gert Rietveld, Ronald van Leeuwen, Dennis Hoogenboom, and Johan Rens, "Characterizing metering errors of static energy meters and their sensitivity to changes in load conditions", submitted to EMC Europe 2019, Barcelona, Spain, September 2019.

[5] CLC TR 50579:2012, "Electricity metering equipment (A.C.) Severity levels, immunity requirements and test methods for conducted disturbances in the frequency range $2 \mathrm{kHz}-150 \mathrm{kHz}$, July 2012 .

[6] CLC TR 50627:2015, "Electromagnetic interference between electrica equipment/systems in the rrequency range below $150 \mathrm{kHz}$ ", November 2015.

[7] CIGRE/CIRED JWG C4.24 TB 719, "Power quality and EMC issues with future electricity networks", March 2018
[8] Directive 2014/30/EU of the European Parliament and of the Council on the harmonisation of the laws of the Member States relating to electromagnetic compatibility, 26 February 2014

[9] Directive 2014/32/EU of the European Parliament and of the Council on the harmonisation of the laws of the Member States relating to the making available on the market of measuring instruments, 26 February 2014

[10] EN 50470-3:2006, "Electricity metering equipment (a.c.), Part 3: Particular requirements - Static meters for active energy (class indexes A, B, and C)", 2006.

[11] IEC 61000-4-19:2014 Testing and measurement techniques - Part 19: Test for immunity to conducted, differential mode disturbances and signalling in the frequency range $2 \mathrm{kHz}$ tot $150 \mathrm{kHz}$ at a.c. power ports.

[12] P.S. Wright, G. Rietveld, F. Leferink, H.E. van den Brom, F.R.I Alonso, J.P. Braun, K. Ellingsberg, M. Pous, and M. Svoboda, "Evaluation of EMI effects on static electricity meters", CPEM Conf. Dig., Paris, France, July 2018.

[13] F. Barakou, P.S. Wright, H.E. van den Brom, G.J.P. Kok, G. Rietveld, "Detection methods for current signals capable of causing errors in static electricity meters", EMC Europe Conf. Dig., Barcelona, Spain, Sept. 2019.

[14] K. Lind, T. Srsdal, and H. Slinde, "Design, modeling, and verification of high-performance AC-DC current shunts from inexpensive components," IEEE Trans. Instr. Meas., vol. 57, no. 1, pp. 176-181, Jan. 2008.

[15] G. Rietveld, C. Kramer, E. Houtzager, O. Kristensen, D. Zhao, C. de Leffe, and T. Lippert, "Characterisation of a wideband digitiser for power measurements up to $1 \mathrm{MHz}$ ", IEEE Trans. Instr. Meas., vol. 60, pp. $2195-2201$ (2011). 